\title{
DETERMINAÇÃO DA UMIDADE RELATIVA DO CAMPO OPERATÓRIO COM ISOLAMENTO ABSOLUTO, MODIFICADO E RELATIVO - ESTUDO PILOTO
}

\author{
DETERMINATION OF RELATIVE HUMIDITY OF THE OPERATIVE FIELD WITH \\ ABSOLUTE, MODIFIED AND RELATIVE ISOLATION - PILOT STUDY
}

\author{
Nicolas Andres Caviglia', Oscar Arauco Urzagaste ${ }^{2}$, Patrícia Correia de Siqueira ${ }^{3}$, Rafael de Almeida Decurcio ${ }^{4}$, Daniel de \\ Almeida Decurcio ${ }^{5}$ \\ 1 - DDS. Associação Brasileira de Odontologia - Seção Goiás \\ 2 - DDS. Arauco Odontologia SRL \\ 3 - DDS, MSc, PhD. Faculdade de Odontologia da Universidade Federal de Goiás \\ 4 - DDS, MSc. Associação Brasileira de Odontologia - Seção Goiás \\ 5 - DDS, MSc, PhD. Faculdade de Odontologia da Universidade Federal de Goiás
}

\section{RESUMO}

Objetivo: Determinar a umidade relativa (UR) do ar ao nível da borda incisal dos incisivos centrais superiores sob diferentes técnicas de isolamento do campo operatório. Material e Métodos: As medições de UR foram realizadas com um termo-higrômetro digital. Quatro pacientes foram selecionados e submetidos a três diferentes técnicas de isolamento: 1- isolamento absoluto; 2- isolamento modificado; 3- isolamento relativo. Nas técnicas de isolamento modificado e relativo foram ainda colocados dispositivos de aspiração por compressor e por bomba a vácuo, para verificação de sua influência na UR. Após o isolamento, o higrômetro foi posicionado ao nível da borda incisal dos incisivos centrais superiores e as medidas de UR foram registradas e calculadas as médias. Resultados: A UR do ambiente no dia das aferições foi de $31 \%$. As medições com isolamento relativo apresentaram média de $98 \%$. O uso de aspiração reduziu os valores de UR para $86 \%$ com compressor e $69 \%$ com bomba a vácuo. As medições realizadas com isolamento modificado apresentaram valores que variaram de $31 \%$ a $95 \%$, dependendo do uso de sistemas de aspiração, enquanto as obtidas sob isolamento absoluto apresentaram um valor médio de $33 \%$. Conclusões: As medições demonstram que a UR se encontra quase totalmente saturada ao nível de incisivos centrais superiores com isolamento relativo, enquanto os valores obtidos com o isolamento absoluto demonstram que é verdadeiramente absoluto, propiciando as melhores condições para os procedimentos clínicos.

Palavras-chave: Umidade do ar, Isolamento absoluto, Dentística operatória, Restauração dentária.

\section{INTRODUÇÃO}

O isolamento absoluto é uma técnica que data do ano de 1864, quando o cirurgião-dentista Sanford Christie Barnum a apresentou na Sociedade Dental de Nova York como uma possibilidade para uso nas técnicas restauradoras da época ${ }^{1}$. Isso ocorreu muito antes do desenvolvimento da adesão e das técnicas e materiais atuais, que determinam a necessidade de sua utilização para procedimentos restauradores.

A Endodontia provavelmente é a especialidade odontológica em que mais se utiliza o isolamento absoluto. O lençol de borracha constitui uma barreira física que minimiza a contaminação do sistema de canais radiculares por micro-organismos do meio bucal e ainda protege a cavidade oral e o trato respiratório do paciente de objetos e substâncias químicas ${ }^{2}$.

Pesquisas têm demonstrado que os procedimentos restauradores adesivos são sensíveis às condições do meio bucal, como a umidade e a presença de contaminantes como sangue, saliva, fluido gengival e lubrificantes das peças de mão ${ }^{3}$. A umidade do ar é um fator constante na prática odontológica, não só pela presença dos fluidos bucais, mas também pelo ar exalado pelo paciente, que provoca uma elevada umidade na cavidade oral, chegando próxima a $100 \%{ }^{4}$. Sendo assim, um adequado isolamento do campo operatório é essencial para o sucesso de longevidade das restaurações adesivas ${ }^{5}$.

Sabe-se que o isolamento absoluto facilita e aumenta a eficácia de vários procedimentos clínicos, já que proporciona um campo operatório seco e livre de contaminação por fluidos e micro-organismos, melhora o campo visual, afasta os tecidos moles, protege o paciente, o operador e a auxiliar, tornando o tratamento mais seguro e eficaz ${ }^{3,5}$.

Estudos que avaliam as taxas de sucesso e fracasso em restaurações indiretas de cerâmica do tipo inlay cimentadas com diferentes sistemas adesivos e cimentos revelam um índice de apenas $4 \%$ de fracasso após um acompanhamento de 4 anos, quando a cimentação é realizada sob isolamento absoluto ${ }^{6}$. Por 
outro lado, restaurações diretas feitas com resina composta sob isolamento absoluto em cavidades classe I e II mostram taxas de sucesso de $75 \%$ e $64 \%$ respectivamente após 17 e 22 anos de acompanhamento ${ }^{7}$.

Browet e Gerdolle (2017) ${ }^{8}$ sugerem que existem três pontos fundamentais que resultam no sucesso do isolamento absoluto: os materiais, a estratégia e a técnica. Esses pontos devem estar completamente compreendidos e aplicados corretamente para se obter a maior qualidade do isolamento.

No entanto, apesar de todos os benefícios, muitos profissionais não usam o isolamento absoluto rotineiramente em sua prática clínica quando realizam procedimentos adesivos, levando-os a trabalhar principalmente com isolamento relativo do campo operatório. Os principais motivos para a não utilização do isolamento absoluto reportados são dificuldade técnica, consumo de tempo clínico e a dificuldade de conseguir contatos interproximais adequados ao realizar restaurações diretas ${ }^{3,5,9}$.

Através da utilização de meios alternativos que oferecem um isolamento parcial como afastadores labiais, roletes de algodão e sugadores de saliva, consegue-se controlar o contato direto da saliva com as superfícies dentárias a serem restauradas, sem levar em conta a umidade relativa do ar gerada pela respiração do paciente, ponto determinante no protocolo adesivo.

O objetivo desse trabalho foi determinar a umidade relativa do ar ao nível da borda incisal dos incisivos centrais superiores sob isolamento absoluto com dique de borracha, isolamento relativo com abridor de boca e técnica de isolamento modificado.

\section{MATERIAL E MÉTODOS}

Para a análise da umidade, utilizou-se um termo-higrômetro digital (DIEHL Thermotron Hygro), instrumento que mede a temperatura e a umidade relativa do ar ambiente.

Foram realizadas diferentes medições de umidade relativa do ar no ambiente do consultório, para então compará-la com a umidade relativa do ar ao nível de incisivos centrais superiores de quatro pacientes submetidos a diferentes tipos de isolamento do campo operatório.

Após o posicionamento do paciente na cadeira odontológica, foram realizadas três diferentes técnicas de isolamento: 1isolamento relativo com afastador labial e roletes de algodão (Imagem 1A); 2- isolamento modificado com lençol de borracha e uma abertura aumentada para todos os incisivos superiores (Imagem 2A); 3- isolamento absoluto com grampos e lençol de borracha (Imagem 3A). Nas técnicas de isolamento modificado e relativo foram ainda colocados dispositivos de aspiração por compressor e por bomba a vácuo (alta potência), para verificação de sua influência na umidade relativa.

Após a realização do isolamento, o higrômetro digital foi posicionado ao nível da borda incisal dos incisivos centrais superiores e os valores de umidade relativa foram registrados. Um total de 12 medições foram obtidas, das quais calculou-se as médias para verificação dos resultados.

\section{RESULTADOS}

Os valores médios encontrados para cada situação clínica estão descritos na Tabela 1.

A umidade relativa do ambiente do consultório no dia das aferições foi de $31 \%$. Os resultados obtidos nas medições realizadas na boca com isolamento relativo apresentaram um valor médio de $98 \%$, demonstrando que o ar a esse nível, só pela respiração do paciente, estava quase totalmente saturado de umidade (Imagem 1B). O uso de aspiração reduziu os valores de umidade relativa para $86 \%$ com compressor e $69 \%$ com bomba a vácuo (Imagens 1C e 1D).

As medições realizadas com isolamento modificado apresentaram valores que variaram de $31 \%$ a $95 \%$ de umidade relativa, dependendo do uso de sistemas acessórios de aspiração (Imagens 2B, 2C e 2D).

As medições obtidas sob isolamento absoluto do campo operatório apresentaram um valor médio de $33 \%$ de umidade relativa, sendo estes resultados muito próximos ao valor de umidade relativa do ambiente (Imagem 3B).
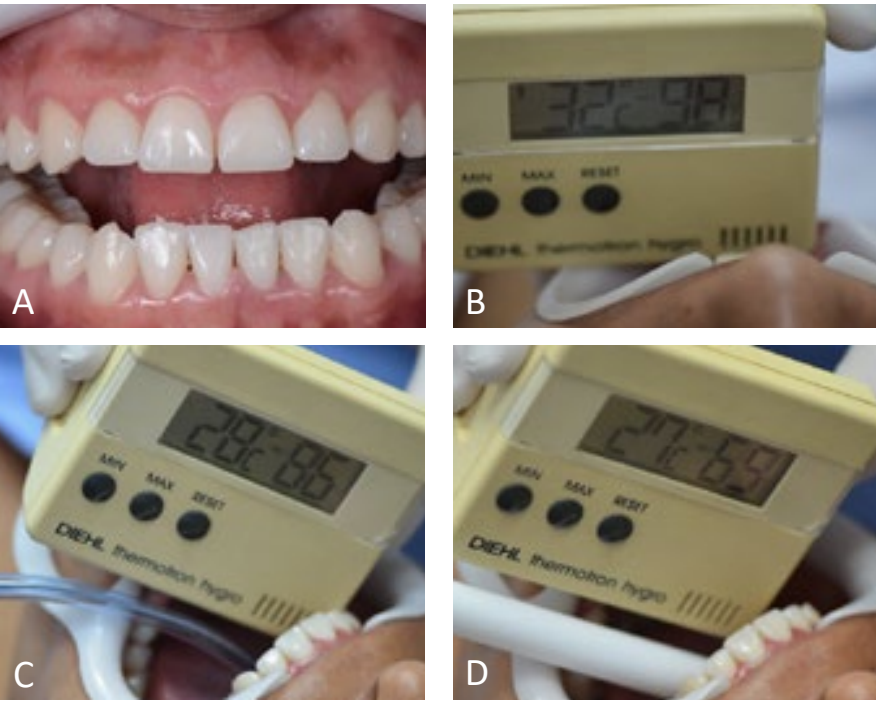

Figura 1 - (A) Aspecto clínico após o isolamento relativo. (B) Mensuração da umidade relativa na região incisal dos dentes anteriores superiores; $(\mathrm{C})$ com dispositivo de aspiração por compressor; (D) com dispositivo de aspiração por bomba a vácuo.
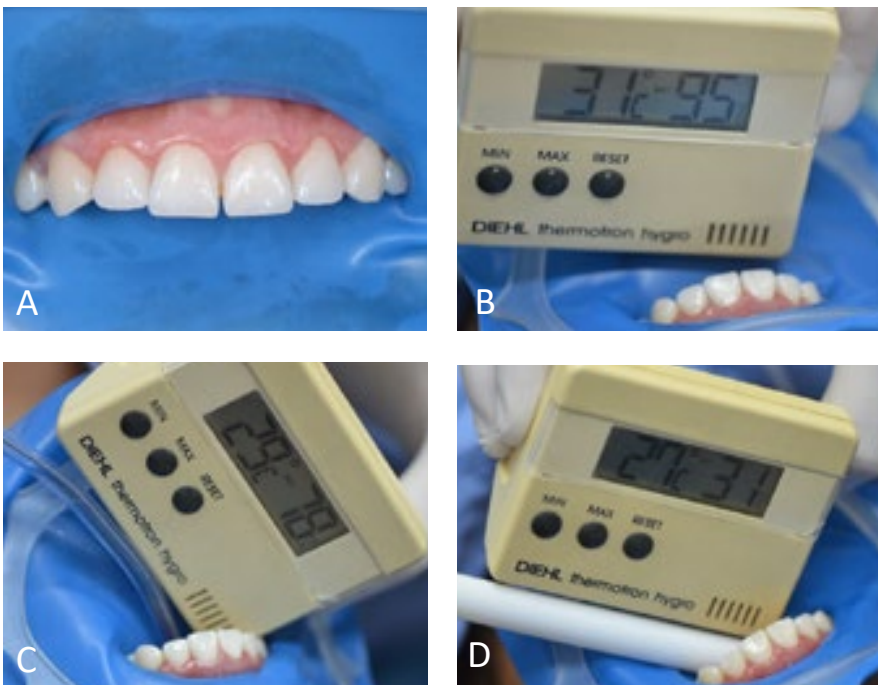

Figura 2 - (A) Aspecto clínico após o isolamento modificado. (B) Mensuração da umidade relativa na região incisal dos dentes anteriores superiores; $(C)$ com dispositivo de aspiração por compressor; (D) com dispositivo de aspiração por bomba a vácuo. 


\section{DISCUSSÃO}

O presente trabalho avaliou a umidade relativa na região de incisivos superiores submetidos a diferentes técnicas de isolamento do campo operatório, e mostrou que o isolamento absoluto e o isolamento modificado associado ao uso de aspirador de alta potência proporcionaram uma umidade relativa próxima à encontrada no ar ambiente.

Os procedimentos adesivos são essenciais para a maioria dos protocolos restauradores usados na odontologia moderna. Diferentes técnicas são usadas para estabelecer uma boa adesão entre os materiais restauradores e o substrato dentário, no intuito de prevenir a formação de gaps e microinfiltrações, reduzindo problemas clínicos e promovendo maior previsibilidade ao tratamento $^{10}$. Esforços significativos têm sido feitos para desenvolver materiais restauradores que aderem adequadamente à dentina no meio bucal sem procedimentos clínicos complicados ${ }^{11}$.

O mecanismo básico da adesão ao esmalte e dentina é essencialmente um processo de troca, que envolve a substituição de mineral removido do tecido dental duro por monômeros resinosos, os quais, após a polimerização, tornam-se interligados micromecanicamente às porosidades criadas. No meio bucal, a água está sempre presente e pode ter um efeito deletério na polimerização, podendo afetar os processos restauradores adesivos de várias maneiras, tanto antes quanto após a polimerização ${ }^{10}$.

Sabe-se que além da umidade, fluidos como saliva, sangue e outros contaminantes são apontados como causas de falhas no processo de adesão. Métodos comuns de controle da umidade incluem lençol de borracha e roletes de algodão combinados com aspiração. O lençol de borracha promove um campo operatório seco ideal enquanto se realiza o procedimento, e esse isolamento é amplamente considerado para reduzir as falhas e aumentar a longevidade do tratamento ${ }^{12}$. Os lençóis de borracha também controlam a infecção cruzada, reduzindo o aerossol microbiano durante o preparo da cavidade e promovendo barreira para potencial propagação de doenças infecciosas no ambiente do consultório odontológico ${ }^{13}$.

A análise das condições de umidade em que se realizará procedimentos adesivos é de extrema importância. Em muitas situações clínicas, nas quais o cirurgião-dentista deve executar ou cimentar restaurações muito próximas aos tecidos moles ou inclusive dentro do sulco gengival, o isolamento absoluto representa para um verdadeiro desafio. No entanto, sabe-se também que este fornecerá sem dúvidas as condições ideais de trabalho. Por isso o conhecimento, os materiais e o treinamento necessários são essenciais para se alcançar um isolamento absoluto do campo operatório que proporcione essas condições.

O sucesso da adesão é desafiado por vários fatores, e pode ser influenciado pelo tipo e tratamento superficial dado ao substrato, o tipo e o tratamento dado ao material restaurador, a correta execução da técnica bem como o tipo e a qualidade do sistema adesivo ou de cimentação utilizado. No geral, os sistemas adesivos são altamente sensíveis à umidade extrínseca à dentina, resultando em valores muito baixos de resistência adesiva em alta umidade, como demonstrado em diversos estudos laboratoriais realizados para avaliar a influência da umidade relativa do meio nos procedimentos adesivos. Plasmans et al. (1993) avaliaram in vitro a sensibilidade à umidade da resistência adesiva de quatro
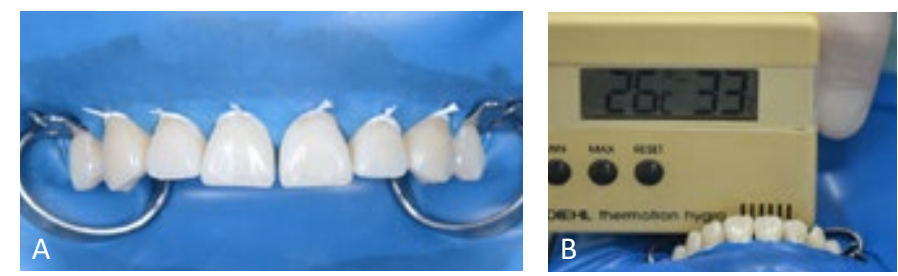

Figura 3 - (A) Aspecto clínico após o isolamento absoluto. (B) Mensuração da umidade relativa na região incisal dos dentes anteriores superiores.

Tabela 1 - Valores médios de umidade relativa obtidos em cada situação clínica analisada.

\begin{tabular}{ll}
\hline SITUAÇÃO CLÍNICA & UMIDADE RELATIVA \\
\hline Ar ambiente & $31 \%$ \\
\hline Isolamento absoluto & $33 \%$ \\
\hline Isolamento modificado & $95 \%$ \\
com aspiração alta potência & $31 \%$ \\
com aspiração compressor & $78 \%$ \\
\hline Isolamento relativo & $98 \%$ \\
com aspiração alta potência & $69 \%$ \\
com aspiração compressor & $86 \%$ \\
\hline
\end{tabular}

sistemas adesivos. Houve diferença significativa para todos os adesivos quando de comparou alta (95\%) e baixa umidade (40\%), tendo os menores valores de adesão em alta umidade.

Miyazaki et al. (2001) $)^{14}$ e Chiba et al. (2004) ${ }^{11}$ investigaram a influência da temperatura e da umidade relativa na adesão à dentina de diferentes sistemas adesivos in vitro. Os autores observaram que quanto maior a umidade, menor a resistência adesiva à dentina. Quando os espécimes foram submetidos à umidade relativa de $95 \%$, a adesão de todos os sistemas avaliados reduziu significativamente. Já a temperatura não influenciou de forma significativa na adesão. Esses resultados sugerem que a umidade extrínseca derivada da cavidade bucal, quando se encontra acima de um nível crítico, pode afetar negativamente a adesão à dentina.

Uma revisão da literatura investigou os resultados de estudos laboratoriais sobre a influência da temperatura e da umidade relativa na adesão ao esmalte e dentina. Observou-se que a umidade é uma variável importante durante os procedimentos adesivos, especialmente quando se testa a resistência adesiva dos materiais in vitro na intenção de simular as condições in vivo. A maioria dos estudos demonstraram a influência da umidade na adesão aos tecidos dentários, mas nenhum estudo explicou adequadamente essa influência nos processos físico-mecânicos que ocorrem na interface. A compreensão do mecanismo da falha adesiva em umidade elevada é fundamental para o desenvolvimento de estratégias racionais para aumentar o desempenho a confiança no uso de adesivos onde essas condições são encontradas. No entanto, os autores ressaltam que a relação entre a evidência do desempenho clínico e os estudos in vitro que avaliam uma propriedade isolada é problemática, mas concluem que o uso do lençol de borracha permanece essencial, mesmo que ele não elimine o problema da alta umidade no ambiente do tratamento ${ }^{10}$. 
Além do isolamento relativo com roletes de algodão e afastadores bucais, outros dispositivos têm sido avaliados para controle da umidade do meio bucal como alternativa ao isolamento absoluto. Kameyama et al. $(2011)^{3}$ avaliaram em pacientes o efeito de dois dispositivos intra-bucais a vácuo (Isolite i2 e Coolex) na temperatura e umidade relativa, comparados ao isolamento absoluto com lençol de borracha e sem isolamento. A umidade relativa e a temperatura foram medidas na região de molares inferiores por um aparelho digital antes e após a colocação dos dispositivos. A umidade relativa na ausência de isolamento absoluto apresentou valores de umidade próximos a 100\%, assim como no presente estudo. Já usando o isolamento com lençol borracha e os dispositivos testados, a umidade passou a ser próxima da detectada no ar ambiente. Os autores concluíram que os três métodos de isolamento testados reduziram efetivamente a umidade relativa do campo operatório, melhorando as condições do ambiente para restaurações adesivas.

Raskin et al. (2000) ${ }^{12}$ realizaram um estudo comparando o desempenho clínico de restaurações posteriores classe I e II em resina composta realizadas com e sem isolamento absoluto. Os pacientes foram acompanhados por um período total de 10 anos e não foram observadas diferenças significativas para os critérios clínicos avaliados quando se utilizou isolamento absoluto ou relativo. Os autores concluíram que o comportamento clínico das restaurações em resina composta de dentes posteriores executadas corretamente usando isolamento efetivo com roletes de algodão e aspiração não foi diferente do comportamento das restaurações realizadas sob isolamento absoluto com lençol de borracha.

Daudt et al. (2013) $)^{5}$ avaliaram o desempenho clínico de restaurações diretas em resina composta em lesões cervicais não cariosas realizadas sob isolamento absoluto e sob isolamento relativo com roletes de algodão. Foram avaliados 62 pacientes e observou-se que o tipo de isolamento não influenciou a performance de restaurações em resina composta classe $\mathrm{V}$ após 1 ano de acompanhamento.

Saraiva et al. (2015) $)^{15}$ avaliaram a temperatura e umidade relativa em sítios intra-bucais anteriores e posteriores e seus efeitos na resistência adesiva em dentina de sistemas adesivos de dois passos. A temperatura e a umidade relativa na região de molares foram maiores $\left(27^{\circ} \mathrm{C}\right.$ e $\left.90,79 \%\right)$ quando comparadas com à região de incisivos $\left(26^{\circ} \mathrm{C}\right.$ e $\left.84,84 \%\right)$. No entanto, essas diferenças não afetaram a resistência adesiva imediata de sistemas adesivos avaliados.

Apesar dos resultados de ensaios clínicos envolvendo desempenho de restaurações em resina composta não evidenciarem diferença substancial entre o isolamento absoluto e isolamento relativo com roletes de algodão, esses estudos são escassos, e tanto resultados de pesquisas in vivo quanto in vitro têm indicado que existe uma influência do meio ambiente na resistência adesiva dos materiais restauradores ${ }^{4,11,14}$, na microinfiltração marginal ${ }^{16}$ e na contração de polimerização de resinas compos$\operatorname{tas}^{17}$. Por isso, é preferível se evitar a realização de procedimentos adesivos na ausência de alguma técnica de isolamento para manter o campo seco ${ }^{3}$.

Além das restaurações diretas, amplamente investigadas, outra condição clínica em que a umidade relativa pode influenciar na adesão são procedimentos de cimentação adesiva de restaurações indiretas de resina ou cerâmica. No entanto, há uma escassez de estudos na literatura que avaliem a influência da umidade do meio no sucesso da cimentação dessas peças. $\mathrm{O}$ critério clínico e a experiência do profissional serão cruciais para diagnosticar se a cimentação sob isolamento absoluto do campo operatório é possível e viável ou se este representará um risco maior que um benefício.

O uso do isolamento absoluto com lençol de borracha ainda se mostra favorável para o desempenho ideal de procedimentos adesivos, confirmando a recomendação tradicional do seu uso para obtenção de condições ótimas de trabalho. Ensaios clínicos controlados e de longo prazo devem ser realizados a fim de se elucidar a real influência da umidade relativa do campo operatório nos procedimentos adesivos da odontologia atual.

\section{CONCLUSÃO}

As medições relatadas neste trabalho demonstram a umidade relativa do ar se encontra quase totalmente saturado ao nível da borda incisal de um incisivo central superior com isolamento relativo do campo operatório. Considerando que essa é a região onde o isolamento relativo teria sua melhor função, deve-se refletir sobre qual seria a condição e controle em outras regiões bucais.

Os valores obtidos com o isolamento absoluto do campo operatório demonstram que é verdadeiramente absoluto, propiciando as melhores condições para os procedimentos clínicos. Deve-se avaliar cada caso clínico individualmente se é possível e viável realizar um isolamento total da estrutura dentária para uma melhor adesão.

\section{REFERÊNCIAS BIBLIOGRÁFICAS}

01. Abrams RA, Drake CW, Segal H. Dr. Sanford C. Barnum and the Invention of the Rubber Dam. Gen Dent. 1982; 30(4):320-2.

02. Malmberg L, Björkne AE, Bergenholtz G. Establishment and maintenance of asepsis in endodontics - a review of the literature. Acta Odontol Escand. 2016; 74(6):431-5.

03. Kameyama A, Asami M, Noro A, Abo H, Hirai Y, Tsunoda M. The effects of three dry-field techniques on intraoral temperature and relative humidity. J Am Dent Assoc. 2011; 142(3):274-80.

04. Plasmans PJJM, Reukers EAJ, Vollenbrock-Kuipers L, Vollenbrock HR. Air humidity: a detrimental factor in dentine adhesion. J Dent. 1993; 21:228-233.

05. Daudt E, Lopes GC, Vieira LCC. Does Operatory Field Isolation Influence the Performance of Direct Adhesive Restorations? J Adhes Dent. 2013; 15:27-32.

06. Krämer N, Ebert J, Petschelt A, Frankenberger R. Ceramic Inlays Bonded With Two Adhesives After 4 Years. Dent Mater. 2006; 22(1):13-21.

07. Ferracane JL. Resin-based Composite Performance: Are There Some Things We Can't Predict? Dent Mater. 2013; 29(1):51-8.

08. Browet S, Gerdolle D. Precision and Security in restorative dentistry: the synergy of isolation and magnification. Int J Esthet Dent. 2017; 12(2):172-185.

09. Rau PJ, Pioch T, Staehle HJ, Döfer CE. Influence of the Rubber Dam on Proximal Contact Strengths. Oper Dent. 2006; 31(2):171-5.

10. Jacquot B, Durand JC, Farge P, Valcarcel J, Périère DD, Cuisinier F. Influence of Temperature and Relative Humidity on Dentin and Enamel Bonding: A Critical Review of the Literature- Part 1: Laboratory Studies. J Adhes Dent. 2012; 14(5):433-46. 
11. Chiba Y, Miyazaki M, Rikuta A, Moore BK. Influence of Environmental Conditions on Dentin Bond Strengths of One-application Adhesive System. Oper Dent. 2004; 29(5):554-559.

12. Raskin A, Setcos JC, Vreven J, Wilson NHF. Influence of the isolation method on the 10-year clinical behaviour of posterior resin composite restorations. Clin Oral Invest. 2000; 4:148-152.

13. Cochran MA, Miller $\mathrm{CH}$, Sheldrake MA. The efficacy of the rubber dam as a barrier to the spread of microorganisms during dental treatment. J Am Dent Assoc. 1989; 119:141-144.

14. Miyazaki M, Rikuta A, Tsubota K, Yunoki I, Onose H. Influence of environmental conditions on dentin bond strengths of recently developed dentin bonding systems. J Oral Sci. 2001; 43(1):35-40.
15. Saraiva LO, Aguiar TR, Costa L, Cavalcanti AN, Giannini M, Mathias P. Influence of Intraoral Temperature and Relative Humidity on the Dentin Bond Strength: An in Situ Study. J Esthet Restor Dent. 2015; 27(2):92-9.

16. Knight GT, Berry TG, Barghi N, Burns TR. Effects of two methods of moisture control on marginal microleakage between resin composite and etched enamel: a clinical study. Int J Prosthodont. 1993; 6(5):475-479.

17. Charlton DG. Effect of humidity on the volumetric polymerization shrinkage of resin restorative materials. Gen Dent. 2006; 54(2):113-116.

\section{ABSTRACT}

Objective: To determine the air relative humidity $(\mathrm{RH})$ at the level of incisal edge of upper central incisors under different techniques of isolation of the operative field. Material and Methods: RH measurements were performed with a digital thermohygrometer. Four patients were selected and submitted to three different isolation techniques: 1- Absolute isolation; 2- Modified isolation; 3- Relative isolation. In the techniques of modified and relative isolation, suction devices by compressor and vacuum pump were also placed to verify their influence on the UR. After isolation, the hygrometer was positioned at the level of the incisal border of the upper central incisors and the RH measurements were recorded and averages were calculated. Results: The $\mathrm{RH}$ of the environment on the day of measurements was $31 \%$.
The measurements with relative isolation showed an average of $98 \%$. The use of suction reduced the $\mathrm{RH}$ values to $86 \%$ with a compressor and $69 \%$ with a vacuum pump. The measurements carried out with modified isolation showed values that varied from $31 \%$ to $95 \%$, depending on the use of aspiration systems, while those obtained under absolute isolation presented an average of $33 \%$. Conclusions: The measurements show that the RH is almost completely saturated at the level of upper central incisors with relative isolation, while the values obtained with absolute isolation demonstrate that it is truly absolute, providing the best conditions for clinical procedures.

Keywords: Humidity, Rubber Dams, Operative Dentistry, Dental Restoration.

\author{
AUTOR PARA CORRESPONDÊNCIA \\ Patrícia Correia de Siqueira \\ E-mail: patricia.csiqueira@hotmail.com \\ Endereço: Faculdade de Odontologia da Universidade \\ Federal de Goiás - Av. Universitária esquina com $1^{\underline{a}}$ \\ avenida, s/n, Setor Leste Universitário, Goiânia-GO. CEP \\ 74605-020.
}

